\title{
Prenatal Exposure to Ethanol Causes Differential Effects in Nerve Growth Factor and its Receptor in the Basal Forebrain of Preweaning and Adult Rats
}

\author{
Francesco Angelucci, ${ }^{1}$ Mauro Cimino, ${ }^{2}$ Walter Balduini, ${ }^{2}$ Luana Piltillo, ${ }^{1}$ and Luigi Aloe ${ }^{1}$ \\ ${ }^{1}$ Institute of Neurobiology, CNR, viale Marx 15, 00137 Rome, Italy; ${ }^{2}$ Institute of Pharmacology and \\ Pharmacognosy, University of Urbino, Vis S. Shiara, 27, 61029 Urbino, Italy
}

\section{SUMMARY}

In this study we investigated nerve growth factor (NGF) levels in the cortex and hippocampus of the offspring of pregnant female Sprague-Dawley rats receiving a single intragastric administration of acute ethanol on the 15th day of gestation and compared them with a control group of rats that received an injection of sucrose. We also examined the distribution of the low-affinity NGF receptor, p75NGFR, on NGF-responsive neurons that are localized in the septum and the nucleus of Meynert, which receive the respective trophic support from the hippocampus and the cortex. In the ethanol-treated group, the results show that at post-natal age 15 days, the NGF septohippocampal pathways were markedly affected. At day 15, the NGF level was significantly higher in the offspring of ethanol-treated rats. By day 40, NGF values in both groups decreased to similar levels. At day 60, however, the NGF level in the ethanol-treated animals decreased to a significantly lower value than that of the control group, which remained essentially unchanged. In parallel, at day 60 the numbers of septal cholinergic neurons expressing p75NGFR were also significantly lower in ethanol-treated rats than in control animals. Because ethanol is known to induce neurological disorders, as well

\footnotetext{
Reprint address:

Luigi Aloe

Institute of Neurobiology

CNR, viale Marx 15,

00137 Rome, Italy

Fax: +06-86-090-370
}

as deficits in cell proliferation and differentiation, the results suggest that one cause of the deleterious effects induced by ethanol is the low availability of NGF during certain stages of postnatal brain development.

\section{KEY WORDS}

NGF, low affinity NGF receptor, cortex, septum, hippocampus, basal forebrain, cholinergic neurons

\section{INTRODUCTION}

In both human and animal systems, alcohol ingestion during pregnancy induces in the offspring several neurobehavioral, biochemical, and molecular changes, resulting in growth retardation $/ 24,26 /$ and brain abnormalities $/ 25,35$ / collectively called "fetal alcohol syndrome" (FAS) /19/. Experimental animal models have shown that the brain regions that are most sensitive to the detrimental effect of ethanol during development are the cerebral cortex $/ 2 /$, hippocampus $16,12,39 /$, septum $/ 32,35 /$, and the cerebellum $/ 21 /$. The effect of ethanol also depends on the dose and on the time of administration. Despite extensive research on FAS in various animal models, the mechanism(s) by which alcohol induces such alterations in brain development is(are) still unclear.

In recent years, several published studies have indicated that neurotrophins regulate growth, differentiation, and behavioral performances $/ 5,14 /$. Biochemical and morphological evidence suggests that without the trophic support of neurotrophins, many neurons would not properly differentiate and survive $15 /$. Among the neurotrophins that have 
been identified $/ 14 /$, nerve growth factor (NGF) is the first and most well-characterized member of a gene family of neurotrophic factors $/ 1,14,22 /$.

NGF is required for the development of selected neurons of both the peripheral and the central nervous systems (CNS) /22,33/ and is essential for maintaining the normal function of cholinergic neurons of the septum and the nucleus of Meynert $/ 18,22 /$, which receive trophic support from NGF produced in the hippocampus and cortex, respectively $/ 18,23 /$. Several studies have demonstrated the functional significance of NGF in basal forebrain cholinergic neurons (BFCN) by showing that during the developmental processes of the CNS, the reduced availability of NGF is detrimental to $\mathrm{BFCN} / 18 /$.

Previous research in our laboratory revealed that chronic, long-term ethanol consumption reduces NGF levels in the hippocampus, as well as the enzymatic activity of choline acetyl transferase (ChAT), a cholinergic enzyme that is regulated by NGF /1/. This study also showed that exogenous NGF administration reverses the neurotoxic effect that is produced by ethanol, but whether alcohol consumption during pregnancy alters brain NGF levels or the expression of NGF receptors in fetal NGF-dependent cells in the CNS is not yet known.

The aim of the present study was to investigate in a rat model whether acute alcohol exposure during a critical period of fetal CNS development (gestation day 15) influences the level of NGF or the expression in BFCN of its low-affinity receptor (p75NGFR) or both. We chose this gestational period for ethanol administration because previous studies have shown that at this time, the developing brain is particularly susceptible to the detrimental effect of ethanol /13/. Furthermore, we used acute rather than chronic ethanol administration because ethanol reportedly causes a greater degree of fetal brain damage when administered in a few high doses rather than in many chronic low doses $/ 9,38 /$.

\section{MATERIALS AND METHODS}

\section{Animals and treatment}

Adult pregnant female Sprague-Dawley rats weighing between $180-250 \mathrm{~g}$ were purchased from
Charles River. The animals were kept on a standard rat food diet and maintained on an artificial 12:12 hr light/dark cycle at a constant temperature of $21 \pm 1^{\circ} \mathrm{C}$. Animal care procedures were implemented according to the intramural committee and institutional guidelines that are in compliance with national and international laws and policies (EEC Council Directive 86/609, OJ L 358, 1, December 12, 1987; Italian Legislative Decree 116/92, Gazzetta Ufficiale della Republica Italiana No. 40, February 18, 1992; NIH Guide for the Care and Use of Laboratory Animals, NIH Publication No. 85-23, 1985).

At day 15 of pregnancy, one group of rats $(n=10)$ received a single administration of intragastric ethanol $(20 \% \mathrm{v} / \mathrm{v}), 4 \mathrm{~g} / \mathrm{kg}$ body weight, under mild anesthesia. A second group of pregnant rats $(n=10)$ were similarly treated, but the ethanol was replaced by an isocaloric equivalent of sucrose ( $4 \% \mathrm{v} / \mathrm{v}, 2 \mathrm{~g} / \mathrm{kg}$ body weight) as a control. This stage of pregnancy was chosen because previous studies have shown that the action of ethanol is most pronounced at this prenatal development stage /13/. At birth, each litter was reduced to eight pups, and the body weight was monitored until the time of sacrifice. At days 15, 40, and 60 of post-natal life, rats from the ethanol group $(n=6)$ and from the sucrose group $(n=6)$ were injected with an overdose of Nembutal. The brains were removed and the frontal, occipital cortex, hippocampus, hypothalamus, and cerebellum were dissected out and immediately stored at $-70{ }^{\circ} \mathrm{C}$ for measuring brain NGF levels.

\section{NGF determination}

Rat NGF was measured using a two-site ELISA, as described in $/ 10,37 /$. Briefly, 96-well polystyrene microtiter immunoplates (Nunc, Roskilde, Denmark) were coated with a mouse monoclonal antibody raised against mouse NGF (2,5 S NGF, clone 27/21, Boehringer Mannheim, Germany). Parallel wells were coated with pre-immune goat IgG to evaluate the non-specific signal, and the plate was incubated overnight at $4{ }^{\circ} \mathrm{C}$. After each step, the plates were washed with washing buffer (Trizma base $50 \mathrm{mM}, \mathrm{NaCl} 200 \mathrm{mM}, \mathrm{NaN}_{3} 0.05 \%$, Gelatin EIA Grade 0.25\%, Triton X-100 0.1\%, pH 7). To block non-specific binding sites, the plates 
were incubated for $2 \mathrm{hr}$ at $37^{\circ} \mathrm{C}$ with $1 \%$ bovine serum albumin (BSA) in coating buffer and washed. NGF standard solutions, ranging from $0-1 \mathrm{ng} / \mathrm{mL}$, were added to each plate. Brain samples were ultrasonicated in the sample buffer at $4{ }^{\circ} \mathrm{C}$, centrifuged for $30 \mathrm{~min}$ at $9.500 \times \mathrm{g}$, and $50 \mu \mathrm{L}$ of supernatant was added to each well. The plate was incubated overnight at $4^{\circ} \mathrm{C}$, washed, and then incubated with monoclonal anti-NGF $(50 \mu \mathrm{L} /$ well $)$ conjugated to $\beta$-galactosidase (Boehringer, Mannheim, Germany) (dil 1:50 in washing buffer $/ 1 \%$ BSA) for $2 \mathrm{hr}$ at $37^{\circ} \mathrm{C}$. After washing, a chromogen substrate (chlorophenol-red-b-D-galactopyranoside, $100 \mu \mathrm{L} /$ well, Boehringer, Mannheim, Germany) was added to each well; incubating the plate for $2 \mathrm{hr}$ at $20^{\circ} \mathrm{C}$ resulted in a colorimetric reaction that was measured at $570 \mathrm{~nm}$ in a Dynatech MR 5000 microplate reader (Limbro Co., Hamden, $\mathrm{CN})$. Specificity for rat NGF was assessed using a recombinant human NGF (Genentech, San Bruno, CA, USA). Recombinant human brain-derived neurotrophic factor is not recognized in the ELISA at concentrations up to $20 \mathrm{ng} / \mathrm{mL} / 10 /$.

\section{Immunohistochemistry}

Ethanol- $(n=4)$ and sucrose-treated $(n=4)$ rats were anesthetized with Nembutal and transcardially perfused with paraformaldehyde in phosphate buffer (PB) ( $\mathrm{pH} \mathrm{7.4).} \mathrm{After} \mathrm{perfusion,} \mathrm{the} \mathrm{brains} \mathrm{were}$ removed and post-fixed for $12 \mathrm{hr}$, cryoprotected in PB containing $20 \%$ sucrose, sectioned at $30 \mu \mathrm{m}$ on a cryostat, and serial brain sections [spanning from the septum (Plate 14) to the nucleus of Meynert (Plate 26), as listed in Paxinos \& Watson /27/], were processed for immunohistochemistry for lowaffinity p75NGFR using a mouse monoclonal antibody raised against rat brain (mAb IgG 192, kindly donated by E.J. Johnson from the Dept. of Pharmacology, Washington University, St. Louis, MO, USA). Free-floating sections were incubated with $\mathrm{mAb}$ IgG 192 overnight at $4{ }^{\circ} \mathrm{C}$. After reacting with peroxidase-labeled anti-mouse IgG, immunocomplexes were processed for immunoperoxidase reactivity using the Vectastain Abc Kit (Vector Lab. Inc., Burlingame, CA, USA), according to the instructions of the manufacturer. Staining specificity was assessed by omitting the primary antibody.
To assess immunoreactivity, we selected anatomically comparable sections and then observed the immunostained neurons with a bright-field microscope at $20 \mathrm{x}$ magnification microscopic images. Quantitative analysis of p75NGFRimmunoreactive neurons and immunoreactivity intensity detected in the medial septum and the nucleus basalis of Meynert was accomplished using a computerized image-analysis system (Axiophot Zeiss microscope equipped with a Vidas Kontron system). The number of p75NGFR-positive magnocellular neurons present in 8-9 matched sections/animal containing the medial septum and the nucleus basalis were measured in experimental and control groups ( $\mathrm{n}=4 \mathrm{rats} / \mathrm{group}$ ). The average values of the pooled cell counts from each group were compared. The immunoreactivity intensity, expressed as grey level values, was determined using standardization criteria across groups (same calibration of the image analysis system, same value of the thresholding for each image, same calibration of the grey scale, ranging from 25-150 arbitrary grey units).

\section{Statistical analysis}

Data are presented as the mean \pm S.E.M. The significance of the differences between the groups were assessed by the Mann-Whitney $U$ test and analysis of variance (ANOVA).

\section{RESULTS}

To evaluate the effect of acute intrauterine exposure to alcohol on the central NGFsynthesizing cells, we used ELISA to measure the levels of this neurotrophin in the anterior and posterior parts of the cerebral cortex and in the hippocampus of the offspring at 15,40, and 60 days of age. Table 1 shows that ethanol treatment did not induce any change in NGF levels in either the anterior or posterior portion of the cerebral cortex, regardless of the stage of brain maturation. Thus, although the amount of NGF decreased with age, no changes were observed between ethanol-treated and control groups. 
TABLE 1

NGF LEVELS IN DIFFERENT BRAIN REGIONS OF RATS TREATED WITH ETHANOL OR SUCROSE ON DAY 15 OF PRENATAL LIFE

\begin{tabular}{cllllll}
\hline \multirow{2}{*}{$\begin{array}{c}\text { Postnatal age }^{1} \\
\text { (days) }\end{array}$} & \multicolumn{2}{c}{ Anterior Cortex } & \multicolumn{2}{c}{ Posterior Cortex } & \multicolumn{2}{c}{ Hippocampus } \\
\cline { 2 - 6 } & Ethanol & Sucrose & Ethanol & Sucrose & Ethanol & Sucrose \\
\hline 15 & $2213 \pm 199$ & $1867 \pm 40$ & $2346 \pm 223$ & $2141 \pm 87$ & $5669 \pm 242^{\mathrm{b}}$ & $4644 \pm 164$ \\
40 & $1327 \pm 175$ & $1234 \pm 135$ & $1141 \pm 87$ & $1256 \pm 216$ & $2718 \pm 183$ & $2847 \pm 224$ \\
60 & $879 \pm 55$ & $1084 \pm 82$ & $1054 \pm 75$ & $1008 \pm 81$ & $2626 \pm 160^{\mathrm{a}}$ & $3156 \pm 180$ \\
\hline
\end{tabular}

${ }^{\text {T}}$ Day of sacrifice. Rat NGF was measured using a two-site ELISA, as described in Materials \& Methods. Results are expressed as $\mathrm{pg} \mathrm{NGF/gram} \mathrm{tissue.} \mathrm{Values} \mathrm{represent} \mathrm{the} \mathrm{mean} \pm$ SEM of 6 replicate animals for each age group and treatment protocol. ${ }^{\mathrm{a}} \mathrm{p}<0.05 ;{ }^{\mathrm{b}} \mathrm{p}<0.01$ (significant difference from sucrose group).

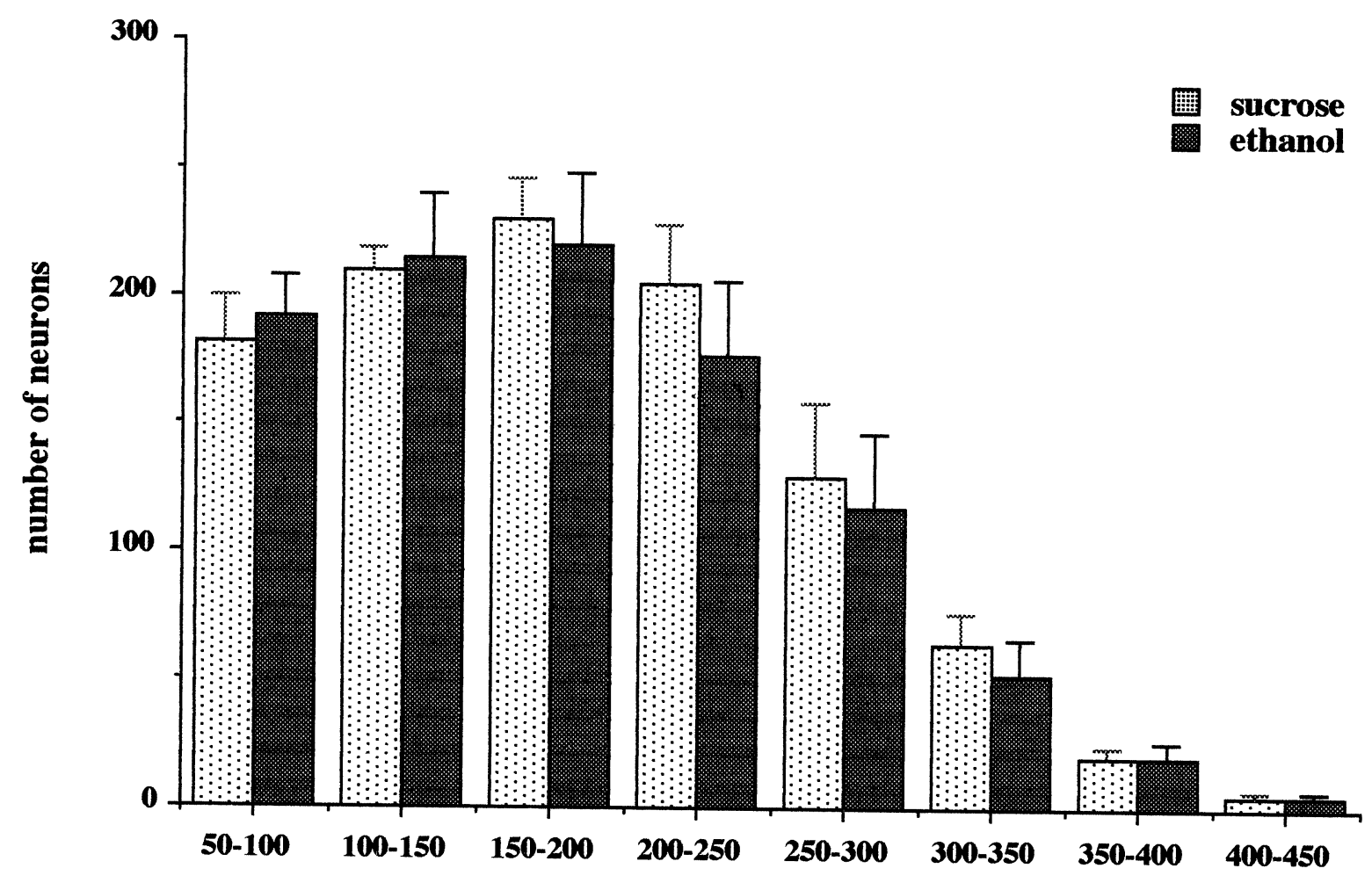

AREA $(\boldsymbol{\mu m} 2)$

Fig. 1. Size and distribution of p75NGFR-immunoreactive neurons in the basal nucleus of Meynert in sucrose- and ethanoltreated rats 2 months after birth. Results are expressed as pg NGF/gr tissue. Values represent the mean \pm SEM of 4 replicate animals per group. No statistical differences were found between groups. 
By contrast, the hippocampus displayed a biphasic pattern of variation of the NGF levels. When compared with NGF levels in the control group, in this brain region we found an ethanolinduced increase in the amount of the neurotrophin at post-natal day 15 , which was no longer present in the hippocampus of 40-day-old animals. At 60 days, however, a statistically significant decrease of the NGF level was observed in ethanol-treated offspring relative to that of the control group.

To determine whether NGF target cells were affected by the ethanol-induced changes in NGF concentration in 60-day-old rats, we carried out an immunohisto-chemical analysis. Serial brain sections from the Meynert basal nucleus and the medial septum were immunostained using an $\mathrm{mAb}$ raised against the low-affinity NGFR. Figure 1 shows the results of a detailed examination of NGFR-positive cell populations in the cerebral cortex, revealing that ethanol treatment did not change the number of neurons expressing the neurotrophin receptor in any neuronal population that was examined in this brain region.

On the other hand, Fig. 2 shows a statistically significant decrease in the number of large (200$350 \mathrm{~m}^{2}$ ) NGFR-immunopositive neurons in the septum of ethanol-treated offspring as compared with the control group. This result was further confirmed by a morphological evaluation of immunostained neurons showing an ethanoldependent reduction in their number and dendritic arborization (Fig. 3).

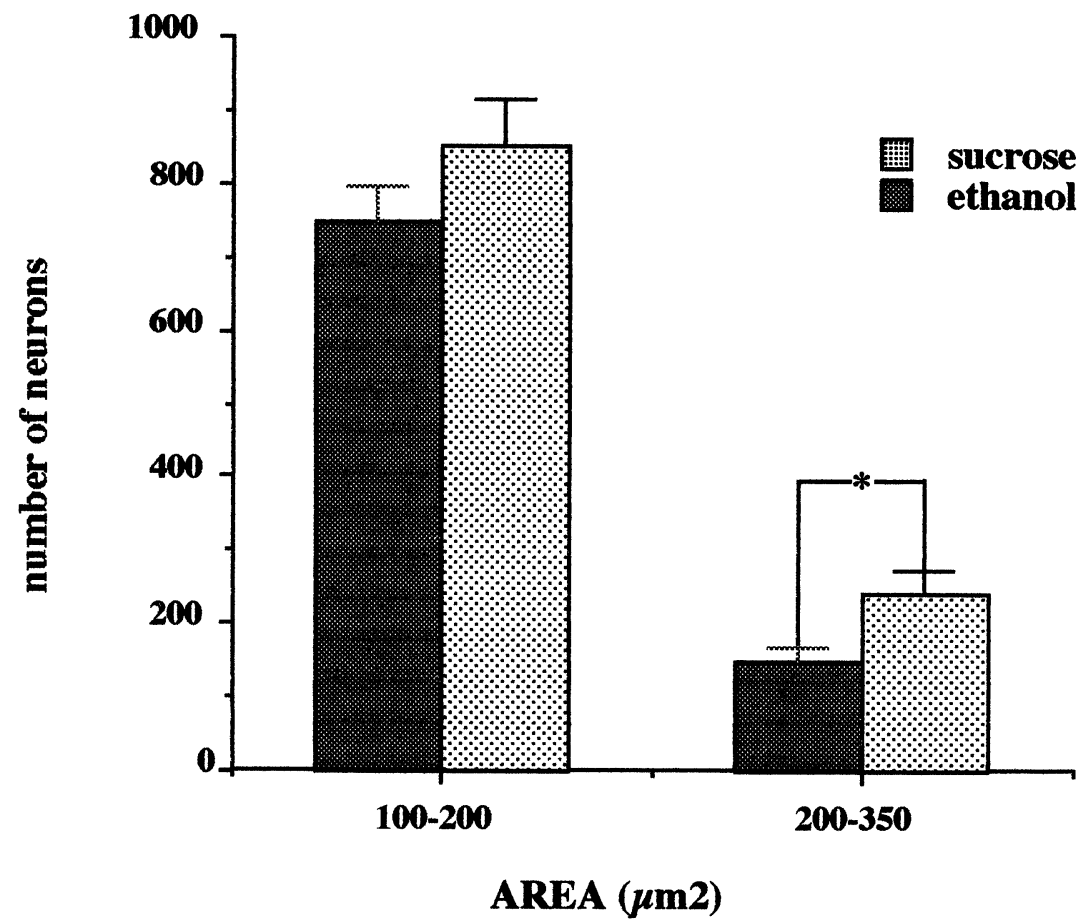

Fig. 2. Septum: Size $\left(\mu \mathrm{m}^{2}\right)$ distribution of p75NGFR-immunoreactive neurons in 2-mo-old rats prenatally treated with sucrose or ethanol on the 15th gestational day. Values represent the mean \pm SEM of four animals per group.

*Significant difference between the groups $(p<0.05)$. 


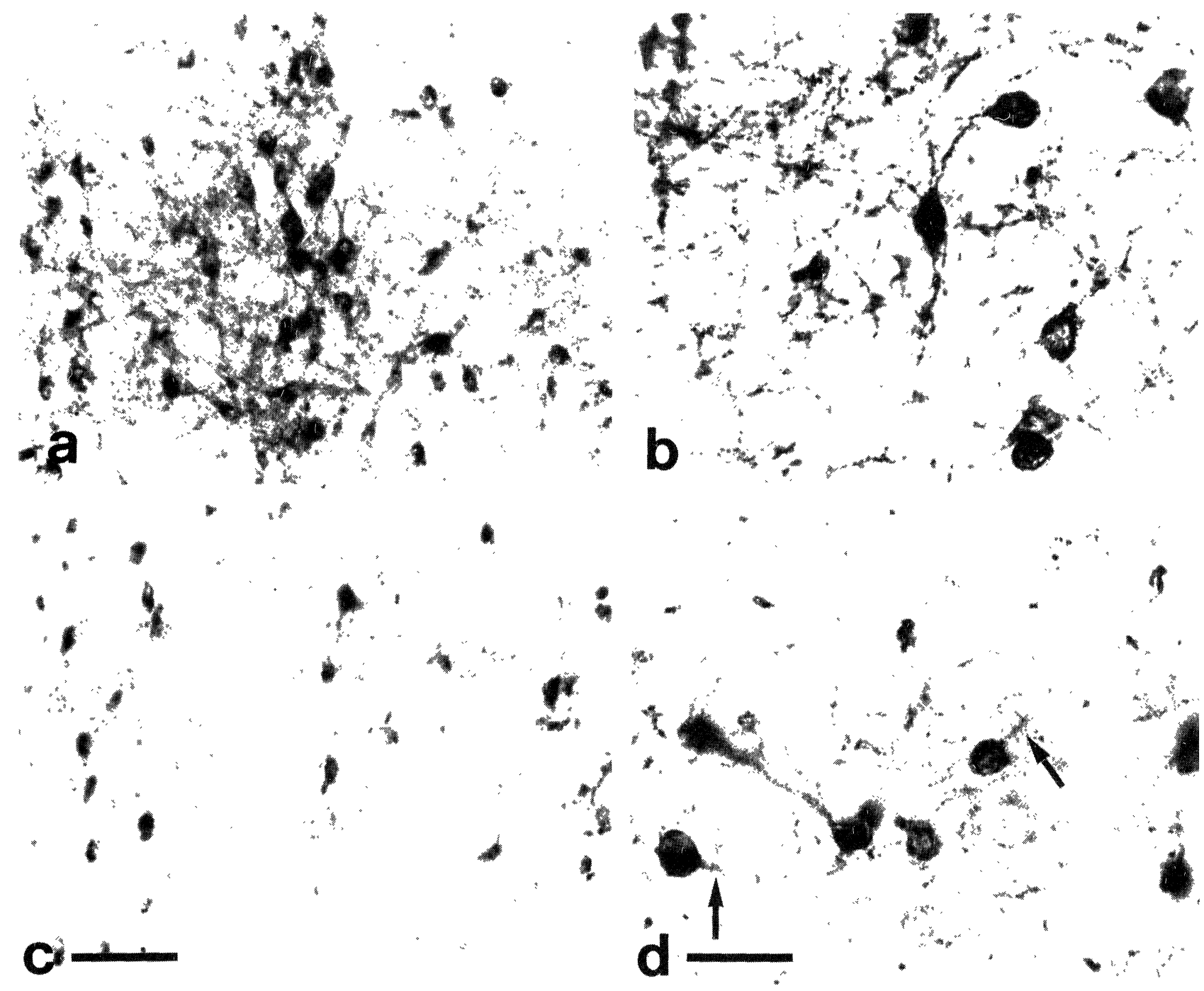

Fig. 3. p75NGFR-immunoreactivity in the medial septum of 2-mo-old rats prenatally treated with sucrose (a,b) or ethanol $(c, d)$ on the 15th gestational day. Note the reduction in the number of cells and in dendritic arborizations (arrows) of the cholinergic neurons in the ethanol-treated animals. Scale bar: (a,c) $100 \mu \mathrm{m}$; (b,d) $50 \mu \mathrm{m}$.

\section{DISCUSSION}

We have previously shown in adult rodents that chronic ethanol intake reduces NGF levels in the hippocampus and the activity of choline acetyl transferase (ChAT) in the septum, hippocampus, and cortex. We have also shown that intracerebral administration of NGF can reduce such toxic effects of ethanol /1/. The effects of ethanol on BFNC are similar to those observed in aging $/ 7,16 /$. The ability of NGF to ameliorate age-related pathologies $/ 18,31 /$ suggests that some of the toxic effects caused by alcohol exposure could be due to an alteration of NGF synthesis and utilization. Whether ethanol consumption during fetal develop-ment affects NGF or NGF-target cells or both in the CNS has not yet been established with certainty.

The results of the present study provide evidence that a single administration of ethanol to a pregnant rat on the fifteenth gestational day is sufficient to alter in the offspring the constitutive level of NGF in the hippocampus, as well as the distribution of p75NGFR in the septum. The same treatment had no effect on the cerebral cortex. In both groups of offspring, the NGF level was high on postnatal day 15 and returned to baseline on day 40 . 
At day 15, the NGF levels in the ethanol-treated group were significantly higher than those in the control group. By day 60 , however, both the NGF levels in the hippocampus and the number of p75NGFR-positive neurons in the septum of ethanol-treated offspring were significantly lower than those in the control group. The concomitant reduction of both markers by 2 months of age in this group clearly suggests that the entire NGF/septo-hippocampal pathway is particularly vulnerable to prenatal exposure to ethanol.

These observations are consistent with the results of Bonthius \& West $/ 9 /$ in a different animal model of FAS showing that hippocampal cell loss is more pronounced when alcohol is administered in several high doses, resulting in high blood ethanol concentrations. Although we did not measure blood alcohol levels in the present study, it is reasonable to assume that the level could have been high enough /4/ to interfere with developing hippocampal neurons, which would have altered the distribution of such cells. Our findings also agree with those of Perez et al. /28/, who demonstrated in newborn rats that a 1-day prenatal exposure to high levels of ethanol reduced the amount of dendritic spines in both the CA1 and CA3 regions of the hippocampus.

Why acute ethanol consumption differentially affects the level of NGF in the hippocampus and the expression of p75NGFR in the septum remains unclear. One explanation for the results could be that the higher NGF concentration found in the offspring on postnatal day 15 resulted from an adaptive response of NGF-producing cells to the toxic effect of ethanol $/ 3,20 /$. This hypothesis is in line with the observation that NGF can counteract the cholinotoxic effect of ethanol both in neuroblast-enriched cultures derived from chick embryo $/ 29 /$ and in aged rats $/ 1 /$. The decreased NGF level on day 60 , on the other hand, might result from a premature degeneration of such cells, a hypothesis that is supported by earlier findings that alcohol intake during pregnancy causes cell loss 19,35/, particularly in hippocampal formation /6/. The immuno-histochemical data in the present study shown in Fig. 2 confirm the results of the latter study.

In both animals and humans $/ 34 /$, evidence has been presented that ethanol intake during pregnancy may result in hippocampal neuronal cell loss. Neurological disorders and behavioral deficits, including psychiatric-related disorders, have also been reported $/ 15,21,30 /$. Alcohol abuse during pregnancy is known to induce a variety of psychiatric disorders in postnatal and adult life $/ 13,28 /$. The hippocampus is a highly plastic brain structure that is involved in memory and learning. Our finding that alcohol exposure in pregnant rats is associated with a modification of endogenous NGF in the hippocampus of the offspring raises the question of whether the decreased availability of this neurotrophin during a critical developmental period is associated with certain neurological deficits in postnatal life. Neurologic deficits resembling certain psychiatric disorders that are associated with a low availability of NGF have also been reported in rats with prenatal-induced cortohippocampal ablation /11/. A decreased availability of NGF or other neurotrophins or both during an early prenatal stage is a possible cause or concause of this deficit $/ 11 /$. This hypothesis agrees with the observation that changes in neurotrophin availability at a critical development stage might be responsible for the pathogenesis of such psychiatric disorders as schizophrenia /17/. As NGF is involved in the growth, differentiation, and survival of certain brain neurons $/ 14,22 /$, it is possible that acute ethanol intake could induce neurological disorders, including schizophrenia /8/. The observation that administration of drugs interfering with neurogenesis also induces changes in neurobehavioral performance in later postnatal age $/ 36 /$ and that schizophrenic patients express a low constitutive level of NGF / / / is in line with this hypothesis.

In summary, the results of this study indicate that in rats, a single prenatal exposure to ethanol induces alterations in NGF levels and in p75NGFRs in the hippocampus of the offspring. The relevance of these findings is two-fold: They highlight the risk that is associated with a single high dose of ethanol during the early stages of pregnancy and indicate the NGF/septo-hippocampal pathway as a potential target for the developmental neurotoxicity of alcohol. Further studies are required to characterize the relevance of both the timing and the exposure model to the interaction between ethanol and this important neurotrophic factor during brain development. 


\section{ACKNOWLEDGMENTS}

The authors wish to acknowledge the invaluable help and encouragement given by Rita LeviMontalcini during this study. This work was supported by the Project on Non-infectious Pathology, Subproject on Behavioral Pathophysiology, by the Italian National Public Health Project "Prevention of risk factors in maternal and child health," Istituto Superiore di Sanità.

\section{REFERENCES}

1. Aloe L, Tirassa P. The effect of long-term alcohol intake on brain NGF-target cells of aged rats. Alcohol 1992; 9: 299-304.

2. Al-Rabiai S, Miller M. Effect of prenatal exposure to ethanol on the ultrastructure of layer $\mathrm{V}$ of mature rat somatosensory cortex. J Neurocytol 1989; 18: 711729.

3. Arendt Th, Brukner MK, Bigl V, Marcova L. Dendritic reorganization in the basal forebrain under degerative conditions and its effects in Alzheimer's disease. II. Aging, Korsakoff's disease, Parkinson's disease, and Alzheimer's disease. J Comp Neurol 1995; 351: 189-222.

4. Balduini W, Costa LG. Effects of ethanol on muscarinic receptor-stimulated phospho-inositide metabolism during brain development. J Pharmacol Exp Therap 1989; 250: 541-547.

5. Barde YA. Trophic factors and neuronal survival. Neuron 1989; 2: 1525-1534.

6. Barnes DE, Walker DW. Prenatal ethanol exposure permanently reduces the number of pyramidal neurons in rat hippocampus. Dev Brain Res 1981; 1: 333-340.

7. Bartus RT, Dean RL, Beer B, Lippa AS. The cholinergic hypothesis of geriatric memory dysfunction. Science 1982; 217: 408-417.

8. Bersani G, Aloe L, Iannitelli A, Maselli P, Alleva E, Angelucci F, Pancheri P. Nerve growth factor (NGF) plasma levels in schizophrenic patients: A pilot study. Am J Psychiatry (in press)

9. Bonthius DJ, West JR. Alcohol-induced neuronal loss in developing rats: Increased brain damage with binge exposure. Alcohol Clin Exp Res 1990: 14: 107-118.

10. Bracci-Laudiero L, Aloe $L$, Levi Montalcini $R$, Buttinelli C, Schilter D, Gillessen A, Ottend U. Multiple sclerosis patients express increased levels of nerve growth factor in cerebrospinal fluid. Neurosci Lett 1992; 147: 9-12.

11. Cimino M, Cattabeni F, Di Luca M, Peruzzi G, Andena ML, Tirassa $\mathrm{P}$, et al. Levels of NGF, p75NGFR, and ChAT immunoreactivity in brain of adult and aged microencephalic rats. Neurobiol Aging
1996; 17: 137-142.

12. Davies DL, Smith DE. A Golgi study of mouse hippocampal CA1 pyramidal neurons following perinatal ethanol exposure. Neurosci Lett 1981; 26:4954.

13. Driscoll CD, Chen J, Riley EP. Passive avoidance performance in rats prenatally exposed to alcohol during various periods of gestation. Neurobehav Toxicol Teratol 1982; 4: 99-103.

14. Ebendal T. Function and evolution in the NGF family and its receptors. J Neurosci Res 1992; 32: 461-470.

15. Falkai $P$, Bogerts B. Cell loss in the hippocampus of schizophrenics. Eur Arch Psychiatry Neurol Sci 1986; 236: 154-161.

16. Gallagher M, Colombo PJ. Aging: The cholinergic hypothesis of cognitive decline. Curr Opinion Neurobiol 1995; 5: 161-168.

17. Hattori M, Nanko S. Association of neurotrophin-3 gene variant with severe forms of schizophrenia. Biochem Biophys Res Comm 1995; 209: 513-518.

18. Hefty $F$. Nerve growth factor promotes survival of septal cholinergic neurons after fimbrial transections. J Neurosci 1986; 6: 2155-2162.

19. Jones KL, Smith DW. Recognition of the fetal alcohol syndrome in early infancy. Lancet 1973; 2: 999-1001.

20. King MA, Hunter BE, Walker DW. Alterations and recovery of dendritic spine density in rat hippocampus following long-term ethanol ingestion. Brain Res 1988; 459: 381-385.

21. Leonard BE. Ethanol as a neurotoxin. Biochem Pharm 1987; 36: 2055-2059.

22. Levi-Montalcini $R$. The nerve growth factor 35 years later. Science 1987; 237: 1154-1162.

23. Lundqvist C, Volk B, Knoth R, Alling C. Long-term effects of intermittent versus continuous ethanol exposure on hipocampal synapses of the rat. Acta Neuropathol 1994; 87: 242-249.

24. Marquis SM, Leichter J, Lee M. Plasma amino acids and glucose levels in the rat fetus and dam after chronic maternal alcohol consumption. Biol Neonate 1984; 46: 36-43.

25. Mena MA, Martin Del Rio R, Herrera E. The effect of long-term ethanol maternal ingestion and withdrawal in brain regional monoamine and amino acids precursors in 15-day-old rats. Gen Pharmacol 1984; 15: 151-154.

26. Messiha FS, Varma SK. Metabolic aspects of fetal alcohol syndrome. Neurobehav Toxicol Teratol 1983; 5: 269-272.

27. Paxinos G, Watson $C$. The rat brain in stereotaxic coordinates, 2nd Ed. London: Academy Press, 1986.

28. Perez HD, Villanueva JE, Salas JM. Behavioral and hippocampal morphological changes induced by ethanol administered to pregnant rats. Ann NY Acad Sci 1991; 625: 300-304.

29. Rahman H, Kentroti S, Vernadakis A. The critical period for ethanol effects on cholinergic neuronal 
expression in neuroblast-enriched cultures derived from 3-day-old chick embryo: NGF ameliorates the cholinotoxic effects of ethanol. Int J Dev Neurosci 1994; 12: 397-404.

30. Roberts GW. Schizophrenia: A neuropathological perspective. Br J Psychiatry 1991: 158: 8-17.

31. Rylett RJ, Williams LR. Role of neurotrophins in cholinergic-neurone function in the adult and aged CNS. Trends Neurosci $1994 ; 486-490$.

32. Schambra UB, Lauder JM, Petrusz P, Sulik KK. Development of the neurotransmitter system in the mouse embryo following acute ethanol exposure: A histological and immunocytochemical study. Int J Dev Neurosci 1984; 8: 507-522.

33. Shelton DL, Reichardt LF. Studies on the expression of the nerve growth factor (NGF) gene in the central nervous system: Level and regional distribution of NGF mRNA suggest that NGF functions as a trophic factor for several distinct populations of neurons. Proc Natl Acad Sci USA 1986; 83: 2714-2718.

34. Shelton DL, Reichardt LF. Expression of the nerve growth factor gene correlates with the density of sympathetic innervation of effector organs. Proc Natl
Acad Sci USA 1984; 81: 7951-7955.

35. Sulik KK, Lauder JM, Dehart DB. Brain malformations in prenatal mice following acute maternal ethanol administration. Int J Dev Neurosci 1984; 2: 203-214.

36. Weinberber DR. Implications of normal brain development for the pathogenesis of schizophrenia. Arch Gen Psychiatry 1987; 44: 666-669.

37. Weskamp G, Otten U. An enzyme-linked immunoassay for nerve growth factor (NGF): A tool for studying regulatory mechanisms involved in NGF production in brain and peripheral tissues. J Neurochem 48: 1779-1786.

38. West JR, Goodlett CR, Bonthius DJ, Hamre KM, Marcussen BL. Cell population depletion associated with fetal alcohol brain damage: Mechanisms of BACdependent cell loss. Alcohol Clin Exp Res 1990; 14: 813-818.

39. West JR, Hamre KM, Cassel MD. Effects of ethanol exposure during the third trimester equivalent on neuron number in rat hippocampus and dentate gyrus. Alcohol Clin Exp Res 1986: 10: 190-197. 

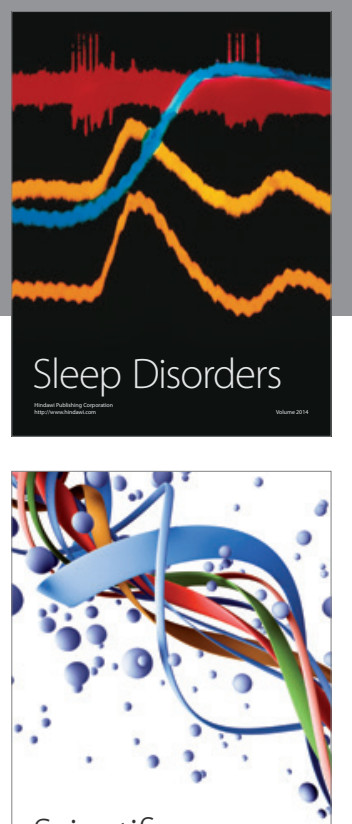

Scientifica
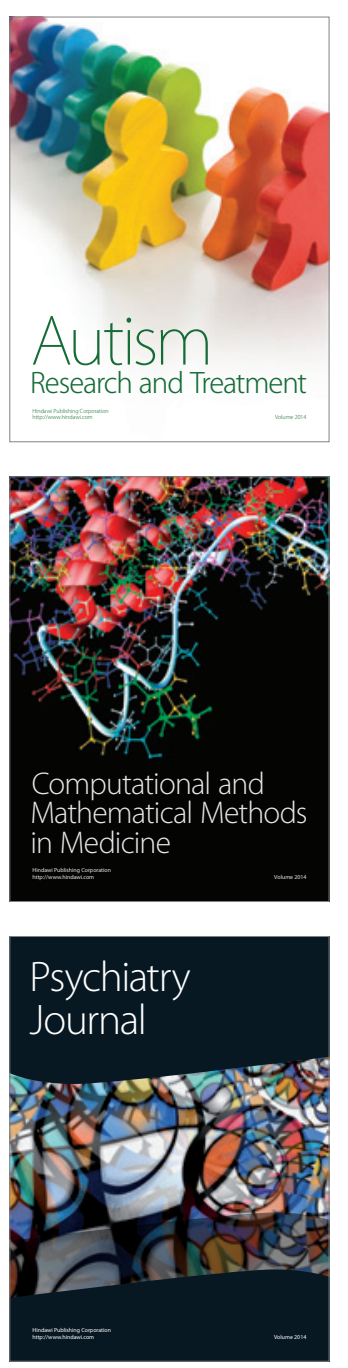
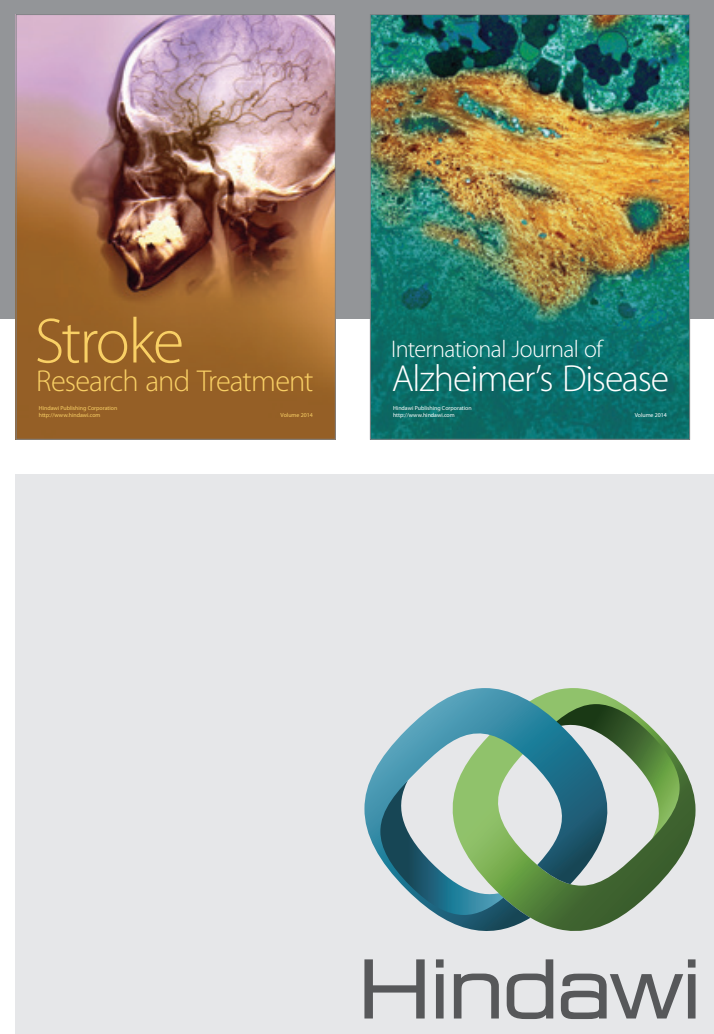

Submit your manuscripts at

http://www.hindawi.com
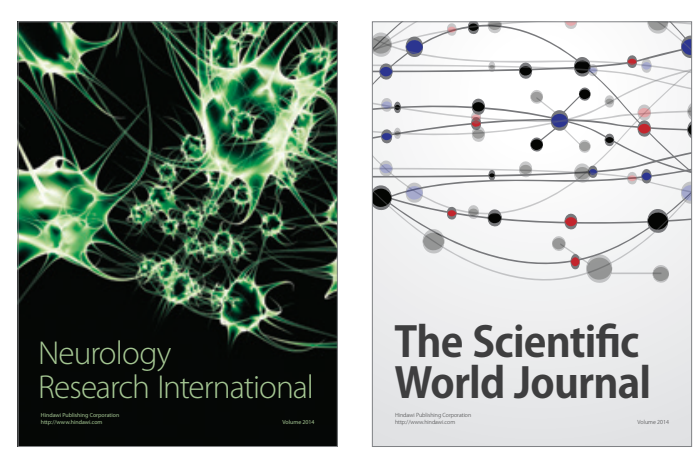

The Scientific World Journal

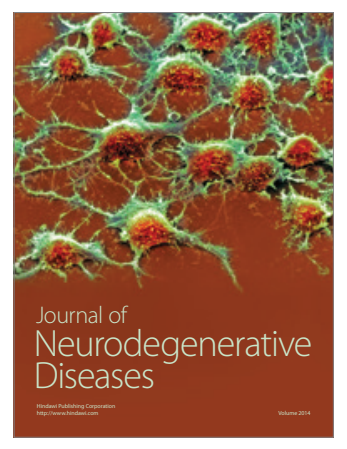

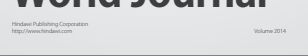

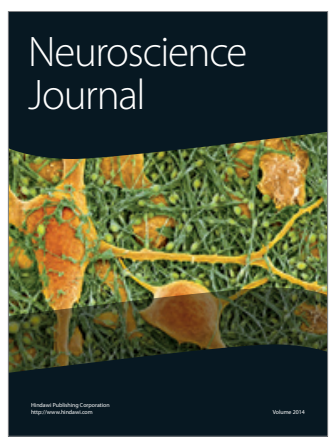

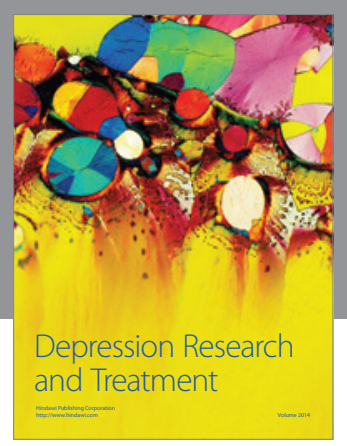
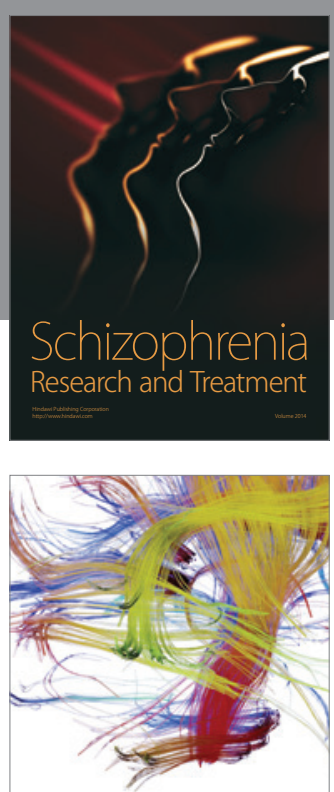

Brain Science

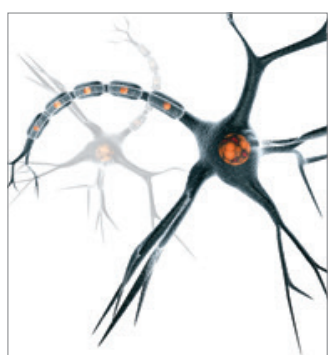

Neural Plasticity
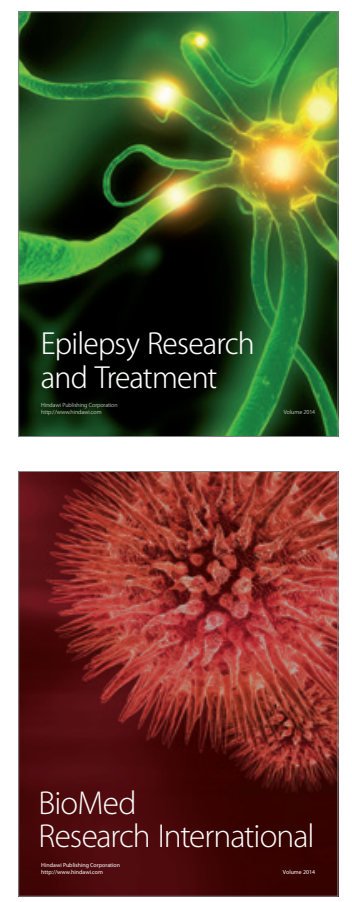

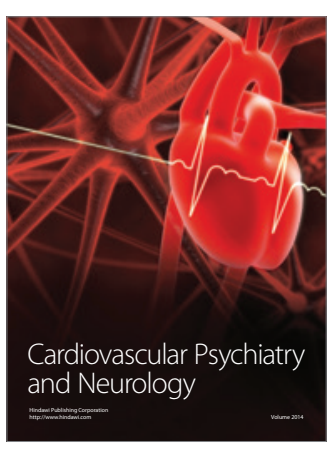

Parkinson's

Disease
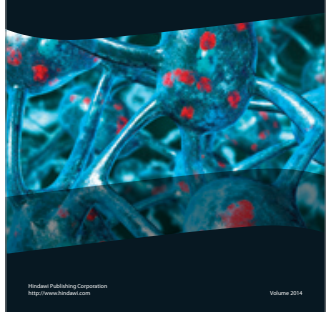